\title{
Combater as alterações climáticas na medicina geral e familiar em Portugal
}

Inês Laplanche Coelho ${ }^{1}$

\section{RESUMO}

O impacto das alterações climáticas já se faz sentir na saúde dos nossos doentes e esta emergência de saúde pública pode vir a pôr em causa a estabilidade do nosso sistema de saúde. As mudanças no clima afetam praticamente todos os órgãos e sistemas, contribuindo para o aumento da incidência e prevalência de diversas patologias. Como médicos de família podemos assumir um papel ativo na mitigação e adaptação às consequências das alterações climáticas na saúde dos nossos doentes, no nosso sistema de saúde e na saúde do nosso planeta através da alteração dos nossos hábitos nas unidades de saúde, da promoção de mudanças de comportamentos e da consciencialização da comunidade em geral para este problema. Somos provavelmente a última geração a poder travar as alterações climáticas e, por isso, temos o dever de agir.

Palavras-chave: Alterações climáticas; Saúde global; Medicina geral e familiar.

\section{INTRODUÇÃO}

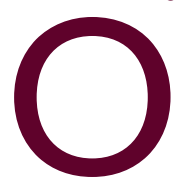

discurso emocionado da ativista ambiental Greta Thunberg na cimeira da Ação Climática das Nações Unidas, em Nova Iorque, alerta-nos para a urgência de agir relativamente às mudanças no clima. As alterações climáticas já estão a ter impacto na saúde dos nossos doentes, nomeadamente através do aumento da mortalidade associada às ondas de calor, ${ }^{1}$ e constituem uma ameaça para a estabilidade do nosso sistema de saúde. Esta já é considerada a maior emergência de saúde pública do nosso tempo e, por isso, devemos agir rapidamente.

Como médicos de família e tendo em conta a nossa vertente preventiva e de âmbito comunitário, podemos preparar-nos para este desafio e ter um papel ativo na mitigação e adaptação às consequências das alterações climáticas na saúde dos nossos doentes, no nosso sistema de saúde e na saúde do nosso planeta em geral.

\section{Alterações climáticas e impacto na saúde}

O impacto das alterações climáticas verifica-se em

1. Médica Interna de Medicina Geral e Familiar. USF Dafundo - ACeS Lisboa Ocidental e Oeiras. Cruz Quebrada, Portugal. diversos órgãos e sistemas e processa-se por diversos mecanismos.

As temperaturas extremas, designadamente as ondas de calor, têm um impacto direto na mortalidade. O calor prolongado sentido no Verão de 2003 na Europa originou um aumento de quatro a cinco vezes das taxas de mortalidade para aquela altura do ano. ${ }^{2} \mathrm{O}$ aumento da temperatura contribui para a elevação das concentrações de ozono e das partículas inaláveis com possível aumento da prevalência de asma e de exacerbações de doenças pulmonares crónicas. ${ }^{3}$ As ondas de calor, por aumentarem o stress térmico e o risco de desidratação, condicionam um aumento de casos de lesão renal aguda e crónica, desequilíbrios eletrolíticos, infeções do trato urinário e nefrolitíase. ${ }^{4}$ Sabe-se que as temperaturas altas e a depleção da camada de ozono, por aumentarem o dano causado pela radiação ultravioleta, aumentam o risco de cancro de pele, incluindo melanoma. ${ }^{5}$ Espera-se que uma elevação de $2{ }^{\circ} \mathrm{C}$ na temperatura do planeta aumente em $10 \%$ a incidência anual de cancros de pele. ${ }^{5}$ Nas áreas urbanas, devido ao efeito de ilha de calor (área urbana que exibe temperaturas mais altas em comparação com o ambiente rural ou suburbano), as pessoas que trabalham ao ar livre durante os períodos mais quentes veem diminuir a sua 
produtividade e aumentar a suscetibilidade às doenças relacionadas com o calor. ${ }^{6}$

As mudanças nos padrões de precipitação alteram a distribuição geográfica e temporal de alguns vetores de doenças, podendo também aumentar a sua atividade, causando maior incidência de patologias como a malária, dengue, doença de Lyme e vírus do Nilo Ocidental, as quais poderão afetar regiões previamente não acometidas por estas doenças. ${ }^{7}$

Os fenómenos climáticos extremos e desastres naturais têm efeitos diretos na saúde mental, através de manifestações sob a forma de ansiedade, depressão ou stress pós-traumático. De acordo com dados da American Public Health Association, sabe-se que $49 \%$ dos sobreviventes do furacão Katrina que atingiu o sudeste dos Estados Unidos da América no Verão de 2005 desenvolveram perturbação de ansiedade e que um em seis desenvolveu perturbação de stress pós-traumático. ${ }^{8}$ A taxa de suicídios no período temporal que se seguiu ao furacão mais do que duplicou. ${ }^{8}$ Para além disso, as alterações climáticas podem originar efeitos indiretos e crónicos por alterarem a forma como nos encaramos a nós próprios e ao mundo que nos rodeia. A solastalgia é o termo utilizado para descrever a angústia ligada à perda do sentido de pertença, consolo e segurança do ambiente físico da pessoa, secundária às consequências das alterações climáticas. ${ }^{9}$

Alguns grupos populacionais são particularmente suscetíveis ao calor, poluição atmosférica e fenómenos meteorológicos extremos, nomeadamente os idosos, grávidas e crianças. ${ }^{10} \mathrm{~A}$ onda de calor ocorrida em Portugal em agosto de 2003 teve um maior impacto no grupo etário de 75 e mais anos onde o excesso de óbitos constituiu cerca de $89 \%$ do total. ${ }^{11}$ No que diz respeito às grávidas, estudos recentes demonstram que extremos de temperaturas aumentam o risco de aborto, parto prematuro, baixo peso ao nascer e mortalidade neonatal em geral..$^{12} \mathrm{~A}$ população pediátrica é particularmente vulnerável pela imaturidade do seu sistema de regulação da temperatura corporal, do seu sistema imunitário e da sua capacidade de destoxificação. ${ }^{13}$ De acordo com a Organização Mundial da Saúde (OMS), 88\% da carga global de doença associada às alterações climáticas recai agora sobre as crianças com menos de cinco anos de idade. ${ }^{14}$

Para além dos impactos diretos na saúde, outras consequências indiretas estão descritas. A mudança no cli- ma ameaça a produtividade agrícola e níveis aumentados de dióxido de carbono diminuem os níveis de proteína, zinco e ferro contidos nas plantas, podendo levar a um aumento na prevalência e gravidade de deficiências nutricionais. ${ }^{10}$ Por fim, a degradação do solo, a escassez de água potável, a pressão populacional e as migrações em massa poderão gerar situações de conflito e violência. ${ }^{15}$

Em Portugal, todos os modelos, em todos os cenários, preveem um aumento significativo da temperatura média em todas as regiões de Portugal até ao fim do século XXI, com um aumento na frequência e intensidade das ondas de calor. ${ }^{16}$ As taxas de mortalidade anuais relacionadas com o calor em Lisboa, que eram de cerca de 6 por 100.000 em 1980-1998, poderão aumentar para um máximo de 29,5 na década de 2050 se nenhuma adaptação ocorrer. ${ }^{17}$ Se o clima se mostrar mais quente e variável, como preveem os modelos, esperam-se aumentos dos níveis de ozono troposférico e de aeroalergénios como os pólenes, com consequente aumento nas exacerbações de doenças respiratórias crónicas como a asma. ${ }^{18}$ Paralelamente, adivinha-se um aumento do risco de incêndios com aumento da prevalência de doenças respiratórias e cardiovasculares por deterioração da qualidade do ar. ${ }^{18}$ Para além dos incêndios espera-se que as cheias também se tornem mais frequentes e este aumento na frequência de desastres naturais poderá ter um forte impacto na saúde mental da população portuguesa. ${ }^{18}$

Os modelos preveem grande variabilidade de precipitação, o que, aliado ao aumento das temperaturas, torna Portugal mais vulnerável a doenças infeciosas transmitidas pela água e alimentos, já que as alterações climáticas favorecem o crescimento dos agentes causais e produção de biotoxinas. ${ }^{18}$ Poderá igualmente aumentar o risco de transmissão de doenças transmitidas por vetores como a leishmaniose, a doença de Lyme e a febre escaro-nodular. ${ }^{17}$

Todos os efeitos descritos podem pôr em causa a estabilidade do nosso sistema de saúde. As alterações climáticas implicam custos associados à maior procura dos cuidados de saúde e necessidade de mais intervenções de saúde pública, custos associados à perda de dias de trabalho e menor produtividade, assim como custos associados à perda de bem-estar. ${ }^{6}$ Prevenir, preparar e gerir os riscos relacionados com o impacto das 
alterações climáticas na saúde é nosso dever enquanto médicos de família.

\section{O que podemos fazer?}

A World Organization of Family Doctors (WONCA) emitiu uma declaração, em abril de 2017, explicando que os médicos de família estão numa posição única para promover os conhecimentos sobre saúde planetária que contribuam tanto para a melhoria da saúde individual, quanto para a do nosso planeta. ${ }^{19}$

Nas nossas unidades podemos começar por implementar a reciclagem, se esta ainda não for realizada. Podemos ter um papel ativo na racionalização dos recursos ao diminuir o consumo de eletricidade, com medidas simples como desligar luzes, desligar os computadores e impressoras ao final do dia e limitar a utilização do ar condicionado. Se for possível ter acesso às contas de eletricidade é possível calcular a pegada carbónica de cada unidade e verificar o progresso alcançado após a implementação destas medidas, motivando, assim, toda a equipa. Podemos diminuir o consumo de papel ao privilegiar a comunicação por $e$-mail, a impressão dos dois lados da folha e evitando a impressão da guia de tratamento das receitas prescritas ou ainda reduzindo a impressão da marcação da próxima consulta, já que muitos utentes preferem anotá-la na agenda do seu telemóvel.

A prescrição terapêutica é responsável por $20 \%$ da pegada de carbono do sistema nacional de saúde inglês. ${ }^{20}$ Reduzir a medicação desnecessária é uma das soluções disponíveis para diminuir o desperdício. ${ }^{20}$ Sabe-se que nos cuidados de saúde primários em Portugal $77 \%$ dos idosos têm, pelo menos, cinco medicamentos prescritos e que $68 \%$ têm, pelo menos, um medicamento potencialmente inapropriado de acordo com os critérios de Beers, havendo aqui um trabalho a fazer por parte dos médicos de família portugueses com impacto positivo para os doentes e para o planeta. ${ }^{21}$ Os inaladores pressurizados são das apresentações farmacêuticas com maior impacto ambiental por conterem hidrofluorocarbonetos que são gases com efeito de estufa. ${ }^{22}$ Assim, devemos preferir, quando possível, a utilização de inaladores de pó seco. ${ }^{22}$

Estas medidas, e muitas outras, estão inscritas no Green Impact for Health Toolkit, uma ferramenta desenvolvida pelo Colégio da Especialidade de Medicina
Geral e Familiar britânico, que visa ajudar os médicos de família a tornarem as suas unidades mais sustentáveis do ponto de vista ambiental, e o qual está acessível para todos. ${ }^{23}$

Com os nossos doentes podemos recomendar mudanças comportamentais que têm benefícios para a saúde do próprio e para a saúde planetária em geral, como o aumento da atividade física ao privilegiar o transporte ativo em vez do transporte motorizado ou a adesão a dietas ambientalmente mais sustentáveis. A alimentação é considerada a alavanca individualmente mais forte para otimizar a saúde humana e a sustentabilidade ambiental na Terra. ${ }^{24}$ Sabe-se que $22,5 \%$ dos portugueses consomem diariamente mais de $100 \mathrm{~g}$ de carnes vermelhas. ${ }^{25}$ Dados do Reino Unido indicam que uma redução no consumo de carne diário (atualmente de 108g) para 50g por dia reduz em $39 \%$ a pegada carbónica. ${ }^{26} \mathrm{~A}$ iniciativa EAT-LANCET propôs uma dieta saudável ambientalmente sustentável, em que recomenda um consumo de carnes vermelhas inferior a $28 \mathrm{~g}$ por dia. ${ }^{24} \mathrm{O}$ Eat Well Guide é outro exemplo de dieta sustentável que garante o aporte recomendado de macro e micronutrientes, assim como de fibras. ${ }^{27} \mathrm{Apli}$ car estas recomendações diminuiria as emissões de gases com efeito de estufa em $31 \%$, a utilização de terreno em $34 \%$, a utilização de água em $17 \%$ e evitaria 17,9 milhões de anos de vida ajustados por incapacidade ao longo da vida da população atual como resultado da diminuição da incidência de diabetes mellitus tipo 2, doenças cardiovasculares e cancro colorretal. ${ }^{26}$

A OMS define um sistema de saúde resiliente ao clima como aquele que é capaz de antecipar, responder, recuperar e adaptar-se a choques e tensões relacionados com o clima para manter a saúde das populações. ${ }^{28}$ Para além das medidas de mitigação faladas, a saúde da nossa população precisa de ser protegida com medidas de adaptação. Durante o Verão está em funcionamento no nosso país um sistema de vigilância diária da mortalidade relacionada com o calor, denominado o Sistema Ícaro, que se articula com o Plano de Contingência para as Ondas de Calor. ${ }^{29}$ Quando se prevê uma onda de calor, este sistema de vigilância emite um alerta vermelho que é posteriormente comunicado às unidades de saúde. Se estivermos atentos a estes alertas, poderemos rapidamente implementar medidas de contingência a nível local e informar os nossos utentes das 
precauções que devem tomar, nomeadamente as populações mais vulneráveis e quem trabalha no exterior. Devemos também estar atentos aos índices de qualidade do ar, como aquele disponibilizado pela Agência Portuguesa do Ambiente (QUALAR), para podermos aconselhar os nossos doentes com doenças respiratórias crónicas a utilizar máscaras de proteção, a ficarem no interior das habitações ou a evitar exercício físico intenso ao ar livre em resposta a alertas de qualidade do ar. $^{30}$ Considerando as doenças infeciosas, deveremos estar mais atentos aos sinais e sintomas das doencas transmitidas por vetores e assegurar uma adequada cobertura vacinal. ${ }^{31}$ Por fim, acredito que podemos ter um papel ativo na consciencialização da comunidade, desenvolvendo ações de formação para profissionais de saúde e material educativo para os nossos doentes e, ainda, defendendo a inclusão de formação nesta área nas faculdades médicas e durante a formação específica em medicina geral e familiar. Deixo ainda o desafio de criar um grupo de estudos nesta área que promova a investigação no âmbito dos cuidados de saúde primários em Portugal.

Somos provavelmente a última geração a poder travar as alterações climáticas e, por isso, temos o dever de agir. Caso contrário, dentro de 20 anos havemos de tentar justificar a nossa apatia para com os nossos filhos e assumir que nada fizemos para salvar o planeta.

\section{REFERÊNCIAS BIBLIOGRÁFICAS}

1. Silva SP, Roquette R, Nunes B, Dias CM. A onda de calor de junho e jutho de 2013: análise dos seus impactes na mortalidade por distrito de Portugal Continental. Bol Epidemiol Obs. 2016;5(15):27-29.

2. Tavares A. O impacto das alterações climáticas na saúde [The impact of climate change on health]. Acta Med Port. 2018;31(5):241-2. Portuguese

3. D'Amato G, Cecchi L, D'Amato M, Annesi-Maesano I. Climate change and respiratory diseases. Eur Respir Rev. 2014;23(132):161-9.

4. Johnson RJ, Sánchez-Lozada LG, Newman LS, Lanaspa MA, Diaz F, Lemery J, et al. Climate change and the kidney. Ann Nutr Metab. 2019;74 Suppl 3:38-44.

5. Kaffenberger BH, Shetlar D, Norton SA, Rosenbach M. The effect of climate change on skin disease in North America. J Am Acad Dermatol. 2017;76(1):140-7.

6. Ebi KL, Hess JJ, Watkiss P. Health risks and costs of climate variability and change. In: Mock CN, Nugent R, Kobusingye O, Smith KR, editors. Injury prevention and environmental health. 3rd ed. Washington: The International Bank for Reconstruction and Development/The World Bank; 2017. P. 153-70.

7. Haines A, Ebi K. The imperative for climate action to protect health. $\mathrm{N}$
Engl J Med. 2019;380(3):263-73.

8. Marti M, Clayton S, Van Susteren L. Making the connection: climate change and mental health [Internet]. American Public Health Association; [s.d.] [cited 2019 Sep 26]. Available from: https://apha.org/-/me$\mathrm{dia} /$ Files/PDF/topics/climate/Climate_Changes_Mental_Health.ashx

9. Albrecht G, Sartore GM, Connor L, Higginbotham N, Freeman S, Kelly $B$, et al. Solastalgia: the distress caused by environmental change. Australas Psychiatry. 2007;15 Suppl 1:S95-8.

10. Salas RN, Solomon CG. The climate crisis: health and care delivery. $N$ Engl J Med. 2019;381(8):e13.

11. Calado R, Nogueira PJ, Catarino J, Paixão EJ, Botelho J, Carreira M, et al. A onda de calor de Agosto de 2003 e os seus efeitos sobre a mortalidade da população portuguesa [The August 2013 heat wave and its effects on the mortality of the Portuguese population]. Rev Port Saúde Pública. 2004;22(2):7-20. Portuguese

12. Kuehn L, McCormick S. Heat exposure and maternal health in the face of climate change. Int J Environ Res Public Health. 2017;14(8):853.

13. Salas RN, Jacobs W, Perera F. The case of Juliana v. U.S.: children and the health burdens of climate change. N Engl J Med. 2019;380(22): 2085-7.

14. Sheffield PE, Landrigan PJ. Global climate change and children's health: threats and strategies for prevention. Environ Health Perspect. 2011; 119(3):291-8

15. Vidal J. Paris climate change talks: what doctors need to know. BMJ. 2015;351:h6316.

16. Agência Portuguesa do Ambiente. O clima em Portugal [Internet].APA; [s.d.] [cited 2019 Sep 9]. Available from: https://apambiente.pt/index. php?ref $=16 \&$ subref $=81 \&$ sub2ref $=118 \&$ sub3ref $=3931$

17. Casimiro E, Calheiros J, Santos FD, Kovats S. National assessment of human health effects of climate change in Portugal: approach and key findings. Environ Health Perspect. 2006;114(12):1950-6.

18. Calheiros J, Casimiro E. Saúde humana e implicações para o turismo. In: Santos FD, Miranda P, editors. Alterações climáticas em Portugal: cenários, impactos e medidas de adaptação. Lisboa: Gradiva; 2006. p. 233-70.

19. WONCA. WONCA statement on planetary health and sustainable development goals [Internet]. WONCA; 2017 [cited 2019 Sep 26]. Available from: http://www.globalfamilydoctor.com/site/DefaultSite/filesystem/documents/policies_statements/Statement\%20on\%20planetary\%20health.pdf

20. National Health Service. Delivering a 'net zero' National Health Service [Internet]. London: NHS; 2020 [cited 2021 Apr 06]. Available from: https://www.england.nhs.uk/greenernhs/wp-content/uploads/sites/ 51/2020/10/delivering-a-net-zero-national-health-service.pdf

21. Simões PA, Santiago LM, Maurício K, Simões JA. Prevalence of potentially inappropriate medication in the older adult population within primary care in Portugal: a nationwide cross-sectional study. Patient Prefer Adherence. 2019;13:1569-76.

22. Janson C, Henderson R, Löfdahl M, Hedberg M, Sharma R, Wilkinson AJ. Carbon footprint impact of the choice of inhalers for asthma and COPD. Thorax. 2020;75(1):82-4.

23. Royal College of General Practitioners. Welcome to green impact for health [homepage]. RCGP; [s.d.] [cited 2019 Sep 26]. Available from: https://www.greenimpact.org.uk/GIforHealth 
24. Willett W, Rockström J, Loken B, Springmann M, Lang T, Vermeulen S, et al. Food in the Anthropocene: the EAT-Lancet Commission on healthy diets from sustainable food systems. Lancet. 2019;393(10170): 447-92.

25. Lopes C, Torres D, Oliveira A, Severo M, Alarcão V, Guiomar S, et al. Inquérito alimentar nacional e de atividade física, IAN-AF 2015-2016: relatório de resultados. Porto: Universidade do Porto; 2017. ISBN 978989-746-181-1

26. British Dietetic Association. Eating patterns for health and environmental sustainability: a reference guide for dietitians [Internet]. BDA 2018 [updated 2021 Nov; cited 2020 Jan 10]. Available from: https://gloucestersustainablefuture.files.wordpress.com/2019/05/bdasustainable-diet-guidelines.pdf

27. The Carbon Trust. The Eatwell guide: a more sustainable diet: methodology and results summary [Internet]. London:The Carbon Trust; 2016 [cited 2020 Jan 10]. Available from: https://www.carbontrust.com/resources/the-eatwell-guide-a-more-sustainable-diet

28. World Health Organization. Operational framework for building climate resilient health systems [Internet]. Geneva:WHO; 2015. Available from https://www.who.int/globalchange/publications/building-climate-resilient-health-systems/en/

29. Instituto Nacional de Saúde Dr. Ricardo Jorge. Ícaro [homepage]. Lisboa: INSA; 2019 [cited 2020 Jan 10]. Available from: http://www2.
insa.pt/sites/INSA/Portugues/AreasCientificas/Epidemiologia/Unidades/UnlnstrObser/Paginas/ICARO.aspx

30. Parker $\mathrm{CL}$, Wellbery $\mathrm{CE}$, Mueller $\mathrm{M}$. The changing climate: managing health impacts. Am Fam Physician. 2019;100(10):618-26.

31. Tavares A. Proteger a saúde das alterações climáticas na Região de Lisboa e Vale do Tejo [Protecting health from climate change in the region of Lisboa e Vale do Tejo]. Rev Port Saúde Pública. 2009; (special no. 25 anos):107-15. Portuguese

\section{CONFLITO DE INTERESSES}

A autora declara não possuir quaisquer conflitos de interesse.

\section{FINANCIAMENTO DO ESTUDO}

A autora não recebeu qualquer financiamento para o desenvolvimento do estudo.

\section{ENDEREÇO PARA CORRESPONDÊNCIA}

Inês Laplanche Coelho

E-mail: ineslaplanchecoelho@gmail.com

http://orcid.org/0000-0002-9693-8823

Recebido em 27-09-2019

Aceite para publicação em 12-02-2020

\section{ABSTRACT}

\section{FIGHTING CLIMATE CHANGE AS GENERAL PRACTITIONERS IN PORTUGAL}

The impact of climate change on health is already being felt and this public health emergency may undermine the stability of our health system. Climate change affects virtually every organ or system contributing to the increase in incidence and prevalence of various pathologies. As general practitioners, we can play an active role in mitigating and adapting to the consequences of climate change on the health of our patients, our health care system, and the health of our planet in general by making changes in our work units, promoting changes in behaviors as well as promoting community awareness of this problem. We are probably the last generation that can stop climate change and therefore we must act.

Keywords: Climate change; Global Health; General practitioners. 